\title{
Changing patterns of investigation and treatment of cardiac failure in hospital
}

\author{
G S Hillis, A Al-Mohammad, M Wood, K P Jennings
}

\begin{abstract}
Objective-To assess the investigation and treatment of cardiac failure in $\mathbf{1 9 9 5}$ and to compare this with management in 1992.

Design-Retrospective consecutive case study.

Setting-University teaching hospital. Subjects-All patients $(n=265)$ discharged from Aberdeen Royal Infirmary in the first quarter (January 1-31 March) of 1995 with a diagnosis of congestive cardiac failure, left ventricular failure, or heart failure (unspecified). These correspond to the International Classification of Diseases 9 th revision codings of 428.0, 428.1, and 428.9 respectively.

Methods-Sociodemographic and clinical data were extracted from the case notes of the above subjects and compared with similar data from the final six months of 1992.
\end{abstract}

Main outcome measures-The use of echocardiography in confirming the diagnosis and delineating the aetiology of heart failure and the use of angiotensinconverting enzyme (ACE) inhibitors in the treatment of patients diagnosed as having heart failure and without contraindications to these agents.

Results-The number of patients discharged in 1995 with a diagnosis including cardiac failure had increased by $55 \cdot 7 \%$ since 1992. The use of echocardiography had also risen from $36.6 \%$ to $72 \%$ (P < 0.0001 ) with an associated increase in the proportion of patients discharged on treatment with an ACE inhibitor $(40 \%$ in $1992 v 55.1 \%$ in 1995: $P<0.001)$. The doses of ACE inhibitors used had also increased significantly $(P<0.001)$. Most patients with cardiac failure continue to be treated by general physicians, who are less likely to use echocardiography $(P<$ $0.01)$ or prescribe an ACE inhibitor $(P<$ $0.05)$ than cardiologists.

Conclusions-There is increasing recognition, more thorough investigation, and improved treatment of heart failure. Despite this there are grounds for concern, both in terms of the adequacy of management and resource implications.

(Heart 1996;76:427-429)

Keywords: cardiac failure; audit; echocardiography; ACE inhibition
The prevalence of heart failure is between $0 \cdot 5-1 \%$ and levels are rising. ${ }^{12}$ This results in considerable morbidity and mortality, plus a rapidly increasing economic cost. ${ }^{3}$ Despite this, cardiac failure remains inadequately investigated and under treated. ${ }^{4-6}$

We assessed the management of patients with cardiac failure in Aberdeen Royal Infirmary in late 1992, and found relatively poor investigation and treatment of the condition. ${ }^{6}$ Since then the management of heart failure has received considerable publicity with wider dissemination of the results of major studies, both in cardiac and non-specialist journals. In addition, we have reported our results at local meetings with colleagues from other medical specialties. In order to assess whether management has improved in the recent past we have re-examined the investigation and treatment of heart failure in the first quarter of 1995 using the same methods as our previous study, thus completing the audit cycle.

\section{Methods}

All patients discharged from the Aberdeen Royal Infirmary during the first three months of 1995 (1 January-31 March) with a diagnosis of congestive cardiac failure, left ventricular failure, or heart failure (unspecified) were identified $(n=265)$. These correspond to the International Classification of Diseases 9th revision codings of 428.0, 428.1, and 428.9 respectively. Those patients ascribed such a diagnosis were included whether or not heart failure was the primary medical problem during their hospital admission. The first hospital admission during which cardiac failure was diagnosed was regarded as the index admission. The units on which patients had been treated were subdivided in a hierarchical fashion. Those managed at any time on a specialist cardiology ward had this recorded as the principal unit on which treatment occurred. A similar strategy was employed for the remaining patients who were under the care of general medical units, leaving a final subgroup who were entirely treated on non-medical wards. The main outcome measures were $(a)$ the use of echocardiography in confirming the diagnosis and delineating the aetiology of heart failure and $(b)$ the use of ACE inhibitors in the treatment of patients diagnosed as having heart failure and without contraindications to these agents. The accuracy of diagnoses was not assessed because the principal aims were to analyse the investigation of cardiac function
Correspondence to:
Dr G Hillis, Department of Medicine and Therapeutics, University of Aberdeen, Aberdeen AB9 2ZD. Accepted for publication 23 May 1996 
and the treatment of heart failure once diagnosed. In addition, it was felt inappropriate to suggest that diagnoses were erroneous on the basis of retrospective case note data. The echocardiographic findings of patients discharged in 1995 were, however, recorded.

Sociodemographic and clinical details were extracted from the case notes of all subjects discharged in 1995 (table) and compared with similar data from the final six months of 1992 (see reference 6 for full details of this cohort). In our original study two patients were excluded as their case notes could not be located and 13 because they were included in the 4th International Study of Infarct Survival. In 1995 all case notes were retrieved and no patients were excluded. Data from the two time points were compared using Fisher's exact and chi-squared tests with Yates' correction and double sided $P$ values used throughout.

\section{Results}

In 1995 an average of 88.3 patients per month were discharged from Aberdeen Royal Infirmary with a diagnosis which included cardiac failure. This represents a $55.7 \%$ increase on the equivalent figure for the last six months of 1992: 56.7 patients per month (including those omitted from subsequent analyses: see above). Most had significant heart failure placing them in New York Heart Asssociation grades III or IV and requiring treatment with loop diuretics (table). In 1992 only $36.6 \%$ of subjects were investigated by echocardiography. By 1995 this had risen to $72 \%$ ( $P<$ $0.0001)$. Of these patients 31 had entirely normal left ventricular function, nine of whom were discharged on an angiotensin-converting enzyme (ACE) inhibitor (none being hypertensive). Forty percent (130/325) of patients with a diagnosis of cardiac failure were discharged on an ACE inhibitor in 1992: in 1995 this had risen to $55.1 \%(146 / 265 ; \mathrm{P}<0.001)$. Twenty six patients who were not so treated had contraindications to ACE inhibition or other recognised reasons whereby this might not be appropriate: these included aortic stenosis $(\mathrm{n}=8)$, significant mitral stenosis $(\mathrm{n}=2)$, combined mitral and aortic stenosis $(n=1)$, renal artery stenosis $(n=4)$, previous adverse reaction ( $n=5$ : severe hypotension $=3, \quad$ rash $=1, \quad$ cough $=1$, , terminal

Comparison of investigation and treatment of patients diagnosed as having cardiac failure in 1995 and 1992 (numbers given in parentheses are percentages)

\begin{tabular}{lll}
\hline & $\begin{array}{l}1995 \\
(n=265)\end{array}$ & $\begin{array}{l}1992 \\
(n=325)\end{array}$ \\
\hline Male/female & $156(58 \cdot 9) / 109(41 \cdot 1)$ & $168(51 \cdot 7) / 157(48 \cdot 3)$ \\
Mean age & 71 years 4 months & 73 years \\
NYHA grade 1 or 2 & $37(14 \cdot 0)$ & $50(15 \cdot 4)$ \\
NYHA grade 3 or 4 & $172(64 \cdot 9)$ & $224(68 \cdot 9)$ \\
NYHA status unclear & $56(21 \cdot 1)$ & $51(15 \cdot 7)$ \\
Treated on cardiac unit & $61(23 \cdot 0)$ & $211(27 \cdot 4)$ \\
Treated on medical ward & $176(66 \cdot 4)$ & $25(7 \cdot 7)$ \\
Treated on other unit & $28(10 \cdot 6)$ & $191(58 \cdot 7)$ \\
Diagnosis purely clinical & $69(26 \cdot 0)$ & $119(36 \cdot 6)$ \\
Underwent echocardiography & $191(72 \cdot 0)$ & $280(86 \cdot 2)$ \\
Discharged on loop diuretict & $225(84 \cdot 9)$ & $130(40)$ \\
Discharged on an ACE inhibitor & $146(55 \cdot 1)$ & $49(15 \cdot 1)$ \\
MI during index admission & $22(8 \cdot 3)$ & \\
\hline
\end{tabular}

« See text.

$t \geqslant 40 \mathrm{mg}$ frusemide or equivalent. illness $(n=5)$, and severe dementia $(n=1)$. After exclusion of these individuals and those patients with normal left ventricular function on echocardiography, 71 patients $(26.8 \%)$ who might have benefited from ACE inhibitors were not thus treated. In 1992, $76 \cdot 2 \%(99 / 130)$ of patients received less than $75 \mathrm{mg}$ captopril, $20 \mathrm{mg}$ enalapril, or an equivalent dose of another ACE inhibitor but by 1995 this had fallen to $54.8 \%(80 / 146$; $\mathrm{P}<$ $0.001)$. Most of patients continue to be treated solely on general medical units (table). The likelihood of undergoing echocardiography remains significantly greater if a patient is treated on a specialist unit (52/61 on cardiac unit $v 139 / 204$ on other unit; $\mathrm{P}<0.01$ ) as does the likelihood of receiving an ACE inhibitor (41/61 on cardiac unit $v$ 105/204 on other unit; $P<0.05)$. However, as noted previously, ${ }^{6}$ the patients treated by specialists tend to be younger (mean age 66 years 11 months if treated by a cardiologist $v 72$ years 8 months if treated by non-cardiac unit). Thus age may be a confounding variable as older patients are less likely to undergo echocardiography (mean age 69 years 7 months if investigated by echocardiography $v 75$ years 11 months if not thus investigated) or receive an ACE inhibitor (mean age 68 years 9 months if recipient of an ACE inhibitor $v 74$ years 5 months if not thus treated).

\section{Discussion}

Although the prevalence of cardiac failure is probably rising ${ }^{12}$ it seems unlikely that this alone could explain the large increase in the frequency of the diagnosis. Instead, the figures probably reflect a growing awareness of the condition with an associated increase in the use of echocardiography particularly by nonspecialists. This is in keeping with published guidelines $^{7}$ and greatly assists the assessment of heart failure. ${ }^{8}$ It does not, however, guarantee appropriate alterations in management.

The major trials demonstrating the effectiveness of ACE inhibition in the treatment of congestive cardiac failure have been associated with considerable publicity. Although this is reflected in an increased use of these drugs there does appear to be room for continued improvement. Many patients who might benefit from ACE inhibitors and who have no obvious contraindications are still not prescribed these drugs. This may be justified in some cases but it appears that many patients are still denied these disease modifying agents. The dosages of ACE inhibitors prescribed have increased, suggesting that clinicians are now more comfortable using these agents and are aware of the recommendations that doses compatible with the major survival studies are used where possible. ${ }^{9-11}$

In summary, we have reassessed the management of cardiac failure in a large teaching hospital, thus completing a cycle of audit. This showed increasing recognition, more thorough investigation, and better treatment of the condition. These results were disseminated locally and there has also been considerable attention 
paid to the management of heart failure in medical journals after the publication of several major trials, particularly those assessing the value of ACE inhibition. We believe it is likely, therefore, that the alterations reflect a generalised improvement in awareness and management. There are, however, no grounds for complacency. Most patients identified had symptoms of severe cardiac dysfunction. It seems likely, therefore, that many patients with milder heart failure are still not being detected while a significant proportion of those with symptomatic disease, and without contraindications, do not receive an ACE inhibitor. On the basis of echocardiography, patients continue to be wrongly labelled as having cardiac failure, demonstrating once again that clinical diagnosis can be very difficult. ${ }^{812}$ Meanwhile, the greater awareness of cardiac failure is placing an increasing strain on services such as echocardiography. Finally, it remains uncertain whether better recognition and treatment of cardiac failure will be maintained if the publicity afforded the condition diminishes. In the long term the emphasis must shift from better treatment towards better prevention. Ultimately the prevalence of cardiac failure will only be reduced through the prevention and optimal management of coronary artery disease plus better control of hypertension.

We are grateful to the medical records staff of Aberdeen Royal Infirmary for their assistance and Mrs Marion Campbell for her statistical advice. This work was supported by grants from the Grampian Area Audit Committee and from Merck Sharpe and Dohme.

1 Sutton GC. Epidemiologic aspects of heart failure. Am Heart f 1990;120:1538-40.

2 McMurray J, McDonagh T, Morrison CE, Dargie HJ. Trends in hospitalization for heart failure in Scotland 1980-1990. Eur Heart $¥$ 1993;14:1158-62.

3 McMurray J, Hart W, Rhodes G. An evaluation of the cost of heart failure to the National Health Service in the UK. of heart failure to the National

4 Clarke KW, Gray D, Hampton JR. Evidence of inadequate investigation and treatment of patients with heart failure. Br Heart F 1994;71:584-7.

5 Editorial. Failure to treat heart failure. Lancet 1992;339. 278-9.

6 Hillis G, Trent R, Winton P, MacLeod A, Jennings $K$. Angiotensin-converting enzyme inhibitors: are we ignoring the evidence? $Q \mathcal{F}$ Med 1996;89:145-50.

7 The Task Force on Heart Failure of the European Society of Cardiology. Guidelines for the diagnosis of heart failure. Eur Heart $\mathcal{F}$ 1995;16:741-51.

8 Francis CM, Caruana L, Kearney P, Love M, Sutherland GR, Starkey IR, et al. Open access echocardiography in $\mathrm{GR}$, Starkey IR, et al. Open access echocardiography in
management of heart failure in the community. $B M \mathcal{F}$ management of

1995;310:634-6.
9 Cohn JN. The prevention of heart failure-a new agenda. N Engl f Med 1992;327:725 7.

10 Cleland JGF, Poole-Wilson PA. ACE inhibitors for heart failure: a question of dose. Br Heart $\mathcal{f}$ 1994;72:106-10

11 Clark AL, Coats AJS. Severity of heart failure and dosage of angiotensin converting enzyme inhibitors. BMF 1995; 310:973-4.

12 Editorial. Clinical signs in heart failure. Lancet 1989;ii: 309-10. 